\title{
Magnetic Cycles and Rotation in Active Late-Type Stars
}

\author{
Ilkka Tuominen ${ }^{1}$, Svetlana V. Berdyugina ${ }^{1}$, and Maarit J. Korpi ${ }^{1,2}$ \\ ${ }^{1}$ Astronomy Division, PO BOX 3000, 90014 University of Oulu, Finland \\ ${ }^{2}$ Laboratoire d'Astrophysique, Observatoire Midi-Pyrénées, 14, Av. \\ Edouard Belin, 31400 Toulouse, France
}

\begin{abstract}
Observational evidence, based both on spectroscopic Doppler imaging and long-term photometry, of strongly nonaxisymmetric spot distributions in magnetically very active late-type stars, with a special cyclic behaviour (the "flip-flop" effect), is presented. Theoretical implications of these results are discussed from the point of view of nonlinear mean-field dynamo theory.
\end{abstract}

\section{Introduction}

Active late-type stars show different type of cyclic behaviour of their magnetic activity than observed in the present-day Sun. This result is based on the analysis of long-term photometric time-series and time sequences of Doppler images. Similar type of behaviour is observed in single giants of FK Com type, RS CVn giants, and active young solar-type stars, the common property being the rapid rotation but small relative differential rotation, thus suggesting that the magnetic dynamo in these stars works in another regime than in the slowly rotating Sun.

\section{Photometric time-series}

The photometric data has been collected by the Phoenix APT, obtained from collaborators, and gathered from literature. We have used two methods to analyse photometry. The first one is to simply locate the photometric minima and plot them as a function of the orbital or mean photometric phase (Berdyugina and Tuominen 1998). A typical example is a RS CVn giant II Peg. There are always two minima separated by half period, forming a slightly inclined line. Sometimes one minimum is stronger, sometimes another one. The strength of the minima does not vary randomly, but a switch, called as the "flip-flop", is observed to happen cyclically, the full length of the cycle being 9.3 years for II Peg.

The result of the second method is illustrated by the analysis of a single solar-like star LQ Hya (Berdyugina, Pelt, Tuominen 2002). Instead of locating the photometric minima, we have now used inversions of the light curve and again plot the longitude of the spot, now as a function of the mean photometric phase. Again we see the same behaviour: there are two spots, separated about half a period, the full "flip-flop" cycle being about 5.2 years. 


\section{Doppler images}

Further evidence that the light curve variations are produced by cool spots has been obtained from a time-sequence of Doppler images (e.g. Berdyugina et al. 1998). Two cool areas are seen at most of the times of observation, the separation in longitude being about $180^{\circ}$, in accordance with the photometric analysis.

We note that no polar caps exist in our Doppler images in II Peg or any other star investigated. In the inversions local line profiles have been calculated from model atmospheres taking into account blends from atomic and molecular lines. The limb darkening both in the continuum and lines is also taken into account (for details see Berdyugina et al. 1998). To compare with the observed profiles in the inversion we have to use additional parameters describing the velocity fields in the stellar atmosphere, namely micro- and macroturbulence, to avoid systematic differences with the observed profile, which may result as artefacts in the images. As a result, the stellar image is obtained as the distribution of effective temperature across the stellar surface, as usual for Doppler imaging.

\section{Differential rotation}

The variations of photometric period can be interpreted as a differential rotation (Hall, 1991, Henry et al. 1995), if the latitude of the spot region varies. An estimate can be obtained from photometry and from Doppler images of LQ Hya (Berdyugina, Ilyin, Tuominen 2001) resulting in $\Delta \Omega / \Omega \approx 0.002$, which is about two orders of magnitude smaller than in the Sun.

In addition, the inclination of the line for II Peg can be interpreted as a sign of differential rotation: if the rotation at lower latitudes of the star is synchronized with the orbital motion, the spot rotation in the higher latitudes may slightly differ from it. The same happens if the spots reflect rotation in the deeper layers, giving estimate for a small radial differential rotation.

It should be noted that the absolute differential rotation $\Delta \Omega$ is more or less constant with a slightly increasing trend towards the more rapid rotation, whilst the relative one $\Delta \Omega / \Omega$ is decreasing about two orders of magnitude.

\section{Dynamo models}

Since the observations indicate that the active stars show highly nonaxisymmetric structures of activity, dynamo models should also include azimuthal direction. Those nonlinear modes to be stable and realised in nature, the differential rotation cannot be large. It is essential that the relative differential rotation is small compared to the absolute one, because the $\alpha$-effect in global mean-field dynamos is proportional to $\Omega$ itself. First steps to this direction have been taken, the nonlinear feedback being only the so called $\alpha$-quenching (Moss, Tuominen, Brandenburg 1991), only large scale Lorentz force with simple dynamics (Moss et al. 1995), or including both (Tuominen et al. 1999). All these models show preference for nonaxisymmetric structure, either symmetric with respect to the equatorial plane or in the third one antisymmeteric with respect to the equatorial plane. Models are still rather simple and do not for example show any cyclic 
variations as the observations. Possibly the nonaxisymmetric and axisymmetric modes should be both stable, the solution being a mixed-mode dynamo. In adddition, there is still no definite observational measurement of the magnetic polarity of the two active regions in the opposite longitudes.

A more extended review has been recently presented by Tuominen, Berdyugina and Korpi (2002) and Korpi and Tuominen (2003).

\section{References}

Berdyugina, S.V., Tuominen, I.: 1998, A\&A 336, L25

Berdyugina, S.V., Ilyin, I., Tuominen, I.: 2001, in The Eleventh Cambridge Workshop on Cool Stars, Stellar Systems, and the Sun, eds. R.J. García López, R. Rebolo, M.R. Zapatero Osorio, ASP, San Francisco, ASP Conf. Ser., 223, CD-1207

Berdyugina, S.V., Pelt, J., Tuominen, I.: 2002, A\&A 394, 505

Berdyugina, S.V., Berdyugin, A.V., Ilyin, I., Tuominen I.: 1998, A\&A 340, 437

Hall, D.S.: 1991, in The Sun and Cool Stars: activity, magnetism, dynamos, eds. I. Tuominen, D. Moss, G. Rüdiger, IAU Coll. 130, Lect. Notes in Physics, Vol. 380, p. 353

Henry, G.W., Eaton, J.A., Hamer, J., Hall, D.S. 1995, ApJS 97, 513

Korpi, M.J., Tuominen, I.: 2003, in Stellar dynamos - perspectives and challenges, Magnetism and activity of the Sun and stars: An international conference in honor of the work of Jean-Louis Leroy, eds. J. Arnaud, N Meunier, EDP Publ. Series Vol 9, 9

Moss D., Tuominen I., Brandenburg A.: 1991, A\&A 245, 129

Moss D.,Barker, D., Brandenburg, A. Tuominen. I.: 1995, A\&A 294. 155

Tuominen, I., Berdyugina, S.V., Korpi, M.J.: 2002, AN 323, 337

Tuominen, I., Berdyugina, S.V., Korpi, M.J., Rönty, T.: 1999, in Stellar Dynamos: Nonlinearity and Chaotic Flows, eds. M. Núnez, A. Ferriz Mas, ASP, San Francisco, ASP Conf. Ser. Vol. 178, p. 195 